International Journal of Distributed and Parallel Systems (IJDPS) Vol.3, No.3, May 2012

\title{
Performance Optimization of WiMAX Mobile Networks with a Predictive Handover Process
}

\author{
M. Grine ${ }^{1}$, A. Najid ${ }^{2,}$ R. Messoussi ${ }^{3}$, \\ ${ }^{1,3}$ Laboratoires des Systèmes de télécommunications et Ingénierie de la Décision \\ « LASTID » FSK Ibn Tofail University Kenitra, ${ }^{2}$ INPT Rabat \\ Most_grine@hotmail.fr

\begin{abstract}
Worldwide Interoperability for Microwave Access (WiMAX) is one of the most promising technologies for the next generation networks as it provides high data rates at medium and long range with full support of mobility. The technology is based on IEEE 802.16 standards and amendments specifying the MAC and PHY layers for fixed, nomadic, portable and mobile access. High speed mobility scenarios require low delay handover so as not to degrade end-to-end QoS indicators, such as delay time or data loss, that are significantly more affected. The WiMAX Forum Network Working Group aims to provide methods for controlled transition of the MS between BSs without significant loss of data or decreased QoS. It has been observed that prediction-based methods reduce handover latency and by the way the packets loss rate. In this paper we use the linear regression model to build a predictive hard handover algorithm that predict the Received Signal Strength Indicator (RSSI) value and advertize the MS to trigger the scanning procedure and the handover process operations reducing so the total handover latency and packets loss rate. The numerical analysis and simulation results show that the proposed method significantly reduces the handover latency and the packets loss rate.
\end{abstract}

\section{KEYWORDS}

WiMAX, NS2, Handover, IEEE 802.16e, prediction, latency, simulation, packets loss.

\section{INTRODUCTION}

The standard IEEE 802.16e [1] provides access to the internet for high speed users anywhere and anytime. It envisage four mobility-related usage scenarios: [2]

1.Nomadic access: provides movement among the cells, but there is no handover support. It means that moving user must establish a new network connection after each cell boarder overrun.

2.Portability and simple mobility fall into a hard handover group. The moving speed is in the range of walking speed and low vehicular speed for portability and simple mobility, respectively. Handover among Base Stations (BS) provide service continuity for all non realtime applications.

3. Simple mobility: The subscriber may move at speeds up to $60 \mathrm{~km} / \mathrm{h}$ with brief interruptions (less than $1 \mathrm{sec}$ ) during handoff.

4.Full mobility comes under the group of a soft handover. Maximal supported speed matches high vehicular speed (about $160 \mathrm{~km} / \mathrm{h}$ ). Handover between BS's provide service continuity for all applications. (Less than 50 ms latency and $<1 \%$ packet loss) is supported.

The WiMAX mobility management architecture was designed to Minimize packet loss, handoff latency, maintain packet ordering to support seamless handover even at vehicular speeds, support both IPv4 and IPv6 based mobility management, support Macro Diversity handover "MDHO" as well as Fast Base Station Switching "FBSS", minimize the number of round trips of signaling to execute handover. [2]

Two types of mobility are supported by the WiMAX network architecture: ASN-anchored mobility (intra-ASN) and CSN-anchored mobility.

The ASN-anchored mobility refers to a scenario where a mobile terminal moves between two base stations belonging to the same ASN while maintaining the same foreign agent at the ASN. The handover in this case utilizes R6 and R8 reference points. 
International Journal of Distributed and Parallel Systems (IJDPS) Vol.3, No.3, May 2012

The CSN anchored mobility refers to an inter-ASN mobility scenario where the mobile station moves to a new anchor foreign agent and the new FA and CSN exchange signaling messages to establish data forwarding paths. The handover in this case is performed via R3 reference point with tunneling over R4 to transfer undelivered packets.

The WiMAX forum has used the framework defined in IEEE802.16e-2005 to further develop mobility management within an end to end network architecture which is designed both on OSI layer 2 (L2) and layer 3 (L3). The L2 handover is also known as micro mobility handover or intra ASN handover [3].

The IEEE802.16e-2005 supports three handover methods:

Hard Handover $(\mathrm{HO})$ : which is the mandatory and the only handover type required to be implemented by the mobile WiMAX initially [4]. The HHO mechanism adopts the Principe of "break before make" which means that the MS will not break the current link until establishment of the connection with the target BS. It results in a sudden connection transfer from one managing BS to a second since the MS can communicate with only one BS at the same time. Consequently, all connections with the serving BSs are broken before a new connection with the target BS is established.

Generally speaking, HHO is the less complex handover type: nevertheless it induces high latency. Consequently, HHO is used mainly for data as it is not suited to real time latencysensitive applications such as VoIP [5].

1. The handover decisions are made by the BS, MS or another entity, based on measurement results reported by the MS. The MS does a radio frequency scan and measures the signal quality of neighboring base stations. The serving BS allocate a scanning intervals for the MS to monitor neighbor BSs and determine the most suitable candidate for handover. Once a handover decision is made, the MS begins synchronization with the downlink transmission of the target BS, performs ranging, if it was not done while scanning, and then terminates the connection with the current serving BS. When the handover process occurs while the MS is active (i.e. the MS exchange data with the serving BS), the undelivered MPDUs at the BS are retained until a timer expires.

2.Face Base Station Switching (FBSS): When the Fast Base Station handover is supported by both the MS and the BS, the MS maintains a list of the neighboring BSs called active set which is continuously monitor by the MS to maintains a valid connection ID with each one of them. However, the MS communicate with only one BS called Anchor BS [3]. When a change of anchor BS is required the connection is switched from one BS to another without having to explicitly perform handoff signaling.

3. Macro Diversity Handover (MDHO) it is similar to FBSS except that the MS communicates, in both up and down link, with all the BSs in the active set called diversity set. The MS needs to monitor the BSs permanently in the Diversity Set then choose an Anchor BS from among them. Moreover, the MS synchronizes and registers to the anchor BS then performs ranging while monitoring the downlink channel for control information.

The two lasts handover methods are optional and are not fully developed yet, however, both of them offer superior performance to HHO.

In this paper, we focus on hard handover (HHO) process.

\section{RELATED WORK.}

In [6] Fattah, Alnuweiri proposes an algorithm which tries to minimize the time spent in handover based on the service flows running at the subscriber station. The algorithm takes into account several aspects of IEEE 802.16e operations such as ranging, authorization, and registration.

The scanning process in [6] has been implemented in detail according to the standard but the SS simply selects those BSs whose RSSI value is higher than a minimum threshold plus a hysteresis value (handover margin). Since the focus lies on the interaction between service flow 
International Journal of Distributed and Parallel Systems (IJDPS) Vol.3, No.3, May 2012

and handover management no novel contributions regarding the scanning mechanisms are made.

In [7] Choi, Hao and Yoo present a group-based scanning scheme which grouping of subscriber stations by signal strength reduces the number of channels to scan so that fast scanning is achieved. To enhance the performance of the group-based scanning scheme, a dynamic neighbor base station list is proposed.

In [8] Hseih et al. proposes an architecture which minimizes the handover latency by using software-based movement tracking technique.

In [4] Akyildiz and Wang, proposes a mobility model characterized not only by historical records but also by stochastic behaviors of mobile users.

In [9] Rouil propose a handover mechanism based on link-going down, which implements channel scanning depending on the level 2 association. The link-going down trigger predicts that the MS will be leaving the coverage area within a certain period of time. The generation of this trigger is based on a measurement algorithm used of link layer performance parameters, such as Signal-to-Interference-and Noise Ratio (SINR), received signal strength indicator (RSSI) and MAC delay. The MS in association level 2 predicts the channel quality and scans channel before the link down. Using link-going down dramatically reduces the handover latency and shows major improvements.

\section{HANDOVER IN WIMAX.}

To support the MS mobility, the IEEE 802.16 e standard provides a handover. When the signal quality of the serving BS worsens due to fading or interference due to mobility, the MS hands over to another BS that provides a better signal quality and quality of service (QoS). The handover procedure in WiMAX is divided into two processes:

\section{Network topology acquisition.}

During this operation, the MS collects information about a channel's description and its physical quality of the BS's neighboring before an actual handover occurs. The network topology information is acquired by performing a Network Topology Advertisement and a scanning process.

\subsection{Network Topology Advertisement.}

According to the IEEE 802.16e standard, the BS should periodically broadcast one MOB_NBRADV message at least every 30s. The message contains the network information such as the number neighboring BSs, cannel information for each neighboring BS, their physical frequency, downlink and uplink channel descriptor (DCD/UCD) messages according to each neighboring BS's identity (BSID).

To make this message the serving BS gathers channel information about each neighboring BS over the backbone. By receiving this message, the MS knows how many BS are in his neighboring and their channel information (i.e. DCD and UCD contents). The MS uses the network topology information in scanning process to facilitate the synchronization with neighboring BSs, because the MS have not to monitor their DCD/UCD broadcasts.

\subsection{Scanning of neighbor's BSs.}

Based on the information, provided by the MOB_NBR-ADV message, the MS becomes aware of the neighboring BSs. The MS monitor their suitability to find a target BS for handover. The MS scan neighboring BSs by exchanging the following messages with the serving BS: 
International Journal of Distributed and Parallel Systems (IJDPS) Vol.3, No.3, May 2012

$>$ MOB_SCN-REQ: sent by the MS to request scanning and negotiate a number of scanning parameters, such as scan duration, interleaving interval, and the number of scan iteration.

$>$ MOB_SCN-RSP: sent by the BS as a response to the MOB_SCN-REQ message, to inform the MS whether it approves or rejects the scanning request. It contains the final scanning allocation parameters and the start frame number for initializing scanning.

$>$ MOB_SCN-REP: transmitted by the MS to report the scanning results, which can be carrier to interference noise ratio (CINR), received signal strength indication (RSSI), relative delay, or round trip delay (RTD). The MS can transmit this message to its serving BS at anytime or at the time indicated in the MOB_SCN-RSP message after each scanning period.

First, an MS receives a MOB_NBR-ADV message and is informed of the existence of the BSs neighboring. If the trigger condition specified in the DCD information is satisfied, the MS sends to the serving BS a MOB_SCN-REQ message to activate a scanning process. This request message contains the following scanning allocation parameters:

$>$ Size of the scanning interval: the period during which the MS can scan for available BS's.

$>$ Size of the interleaving interval: it indicates the period during which the MS can operate normally and can receive/send data from/to its serving BS.

$>$ Number of scan iterations determines how many times the scanning and interleaving intervals are repeated during the total scanning period.

If the BS receives the MOB_SCN-REQ message, it responds with an MOB_SCN-RSP message. The MOB_SCN-RSP message can either grant a scanning interval that is at least as large as that which the MS requests or reject the scanning request by setting the value of scan duration to zero.

After receiving the MOB_SCN-RSP message that approves the scanning request, the MS starts to scan for neighboring BSs at the start frame and continues throughout the scanning interval specified in the response message.

When a neighboring BS is identified by scanning, the MS attempts to synchronize with its downlink transmissions and estimates the quality of its physical channel.

After the end of each scanning interval, the MS may issue the MOB_SCN-REP message to report the scanning results. The serving BS should buffer incoming data addressed to the MS during the scanning interval and forward those data after the scanning interval during any interleaving interval or after completing the scanning operation. These scanning and interleaving intervals are repeated alternately for the number of scan iterations specified.

\section{Basic Handover Operation.}

The handover process begins with a decision that originates either from the MS or the BS (The BS can force the MS to conduct handover) [10]. When a handover occurs, it observes the following procedures: [11]

$>$ Cell reselection: the MS uses the information acquired from an MOB_NBR-ADV message and the serving BS's scheduled scanning intervals to perform cell reselection. It makes a scan to determine the availability and suitability of the BSs neighboring as an HO target.

$>$ Handover decision and initiation: a handover is initiated by a decision to handover from a serving BS to a target BS. The MS can initiate HO by transmitting an MOB_MSHO-REQ message which is acknowledged by the BS MOB_BSHO-RSP message. If the serving BS receives the MOB_MSHO-REQ message or judges that the MS needs to perform a $\mathrm{HO}$, it sends an $\mathrm{HO}$ notification message containing the MS information to one or more potential target BSs over the backbone network to notify that the MS intends to HO. If the serving BS receives an $\mathrm{HO}$ notification response from the target BSs, it selects a target $\mathrm{BS}$ suitable for the MS's HO according to the status of the response message (accept or reject), and then sends an HO confirm message to the selected target BS. Thereafter, the serving BS informs the MS of the selected target BS by sending the MS the MOB_BSHO-RSP message (in the case of MSinitiated HO) or the MOB_BSHO-REQ message (in the case of BS-initiated HO). If the MS receives the MOB_BSHO-RSP or MOB_BSHO-REQ message, it makes a final HO decision 
and sends an MOB_HO-IND message. The MOB_HO-IND message notifies the serving BS of the final decision, which can be a serving BS release, $\mathrm{HO}$ cancellation, or $\mathrm{HO}$ rejection.

$>\mathrm{HO}$ cancellation: The MS can cancel the current $\mathrm{HO}$ at any time, regardless of whether it was the MS or BS that initiated the HO. This cancellation is made by transmitting the MOB_HOIND with the HO cancel option. When the serving BS receives the MOB_HO-IND with the $\mathrm{HO}$ cancel option before the resource retain timer expires, the MS and serving BS resume normal communication. If an MS wants to attempt to handover to a different BS, whether or not that BS was included in MOB_BSHO-RSP or MOB_BSHO-REQ, it requests the serving BS to reject its current $\mathrm{HO}$ instruction by sending an MOB_HO-IND with the HO reject option.

$>$ Synchronization to target BS downlink: To connect with the target BS, the MS synchronizes with the downlink transmissions of the target BS and obtains downlink (DL) and uplink (UL) transmission parameters. If the MS had previously received an MOB_NBR-ADV message including target BSID, physical frequency, DCD, and UCD, this synchronization process can be shortened. Otherwise, the MS synchronizes with the target BS by scanning the possible channels of DL frequency band until it finds a valid DL signal.

$>$ Use of scanning and association results: An MS scans the target neighboring BSs and has the option to try association. If the target BS has previously received HO notification over the backbone from the serving BS, the target BS can place a fast ranging information element (Fast_Ranging_IE) in the UL-MAP to allocate a non-contention-based initial ranging opportunity. Therefore, the MS can use the non-contention-based initial ranging opportunity by scanning the UL-MAP of the target BS for fast $\mathrm{HO}$ ranging process.

$>$ Ranging: An MS and a target BS conduct an initial ranging or $\mathrm{HO}$ ranging after the synchronization with the target BS downlink. An MOB_BSHO-REQ or MOB_BSHO-RSP message informs the MS of the common time interval at which the dedicated initial ranging transmission opportunity for the MS will be provided by the target BS. Therefore, the MS can receive the Fast_Ranging_IE in the UL-MAP of its target BS, which includes a noncontention-based initial ranging opportunity. If the MS confirms that initial ranging opportunity, it can transmit an RNG-REQ code to the target BS without access collision. This operation enables fast ranging because the target BS provides a dedicated UL resource for the ranging request.

$>$ Termination with the serving BS: If the MS decides to carry out an $\mathrm{HO}$ after receiving an MOB_BSHO-RSP or MOB_BSHO-REQ message, the MS terminates service with the serving BS. This operation is accomplished by sending an MOB_HO-IND message with the option of serving BS release. If the BS confirms the release of its service, it starts the resource retain timer. Until the resource retain timer expires, the serving BS retains the MS connections, MAC state machine, and packet data associated with the MS for service continuation. When the resource retain timer expires, the serving BS releases all information about the MS and the MS is disconnected from its serving BS.

$>$ Drops during HO: A drop occurs when an MS has ceased to communicate with its serving BS before the normal HO procedure has been completed. An MS can detect a drop by its failure to demodulate the DL, or by the failure of the periodic ranging mechanism. When the MS has detected a drop during network re-entry with a target BS, it attempts network re-entry with its preferred target BS by the cell reselection procedure. At this time, the MS can try to resume communication with the serving BS by sending an MOB_HO-IND message with the HO cancel option.

$>$ Network entry/re-entry: An MS starts to perform network entry procedures with a new BS after a successful ranging process. If the MS has sent an RNG-REQ that includes a serving BSID during the ranging process, a target BS may request information about the MS from the serving BS over a backbone network, and the serving BS may respond with the requested information, to expedite the network entry process. Therefore, the process of network entry with the target BS can be shortened if the target BS obtains information about the MS from the original serving BS. Depending on the amount of that information, the target BS can 
International Journal of Distributed and Parallel Systems (IJDPS) Vol.3, No.3, May 2012

decide to skip one or more steps among the following network entry procedures: negotiate basic capabilities, privacy key management (PKM) authentication phase, traffic encryption key (TEK) establishment phase, and registration.

The figure 1 shows the operations of the network topology advertisement and scanning procedure while the figure 2 shows the basic Handover operations.

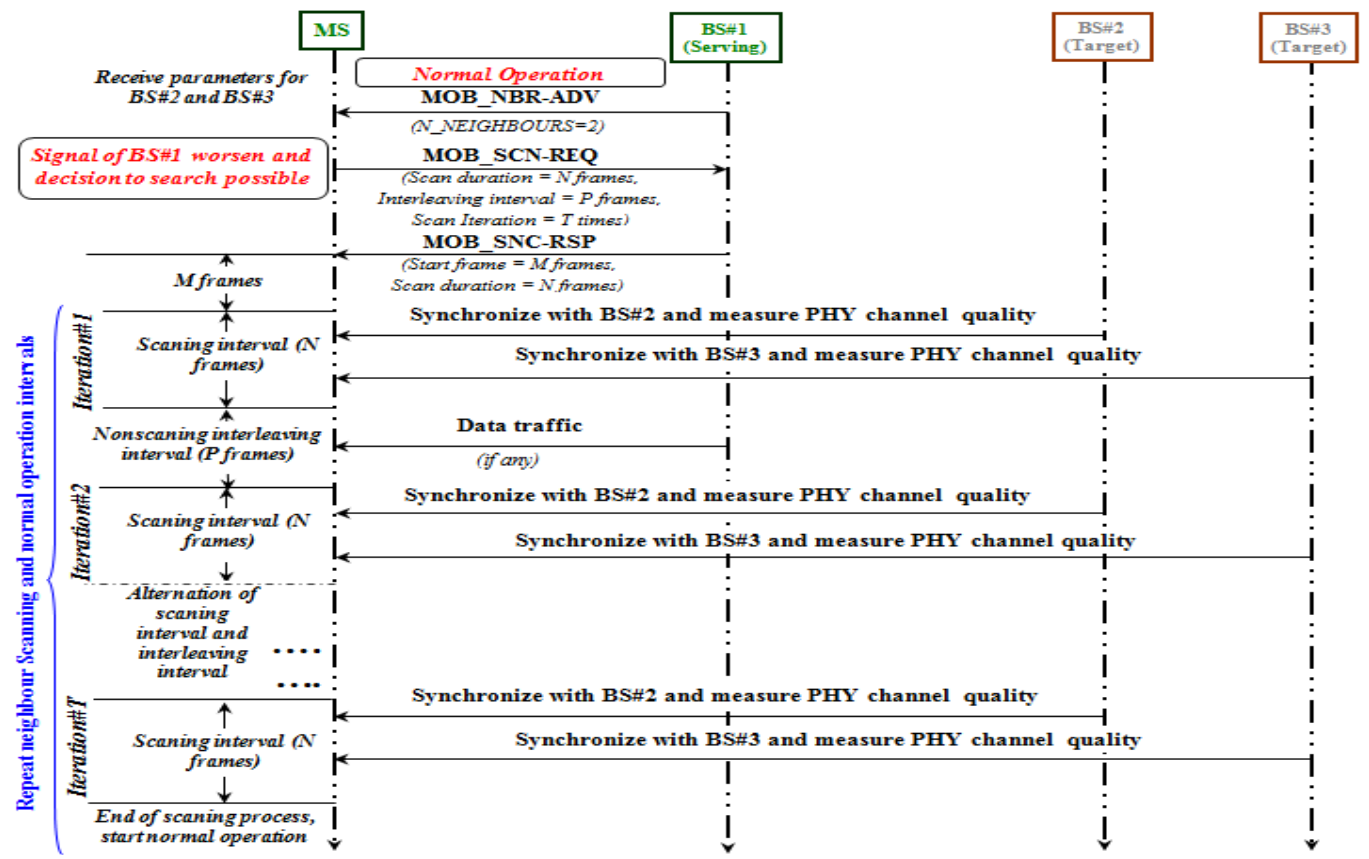

Figure.1. Operation of network topology advertisement and, scanning procedure.

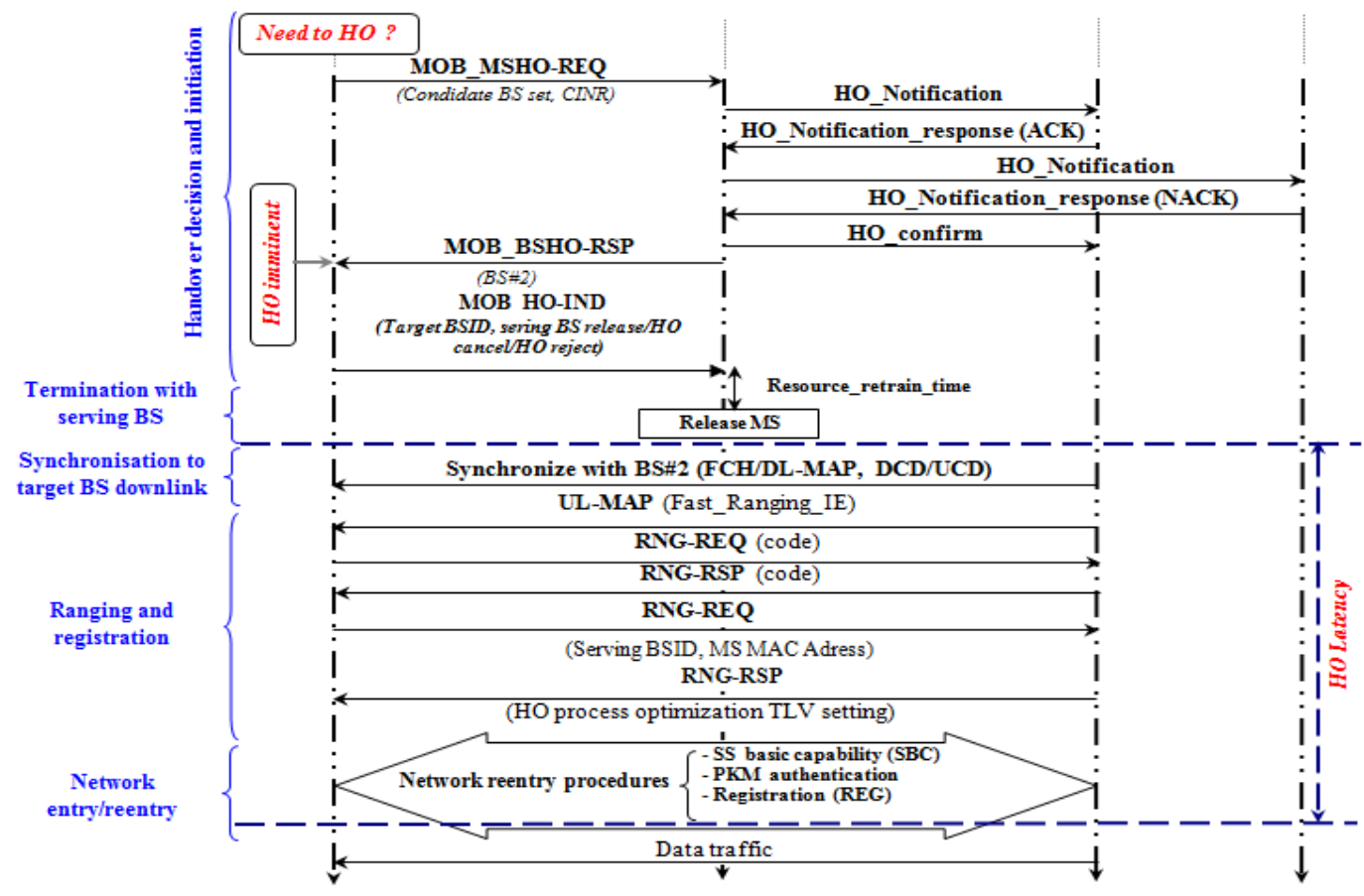

Figure.2. Basic Handover operations. 


\section{PREDICTIVE HARD HANDOVER ALGORITHM}

In this section we describe our contribution to optimized hard handover algorithm. Since it has been observed that prediction-based methods reduce handover latency and by the way the packets loss rate. [12] We us the linear regression model to build a predictive hard handover that predict the Received Signal Strength Indicator (RSSI) value in order to advertize the MS to trigger the scanning procedure and by the way the handover process operations in order to reduce the total handover latency and packets loss rate.

When the serving BS (RSSI)Serving worsen and becomes less than the scanning threshold "Scth" the MS initiate a scanning neighbor BS's to determine their suitability. The MS obtains, periodically through the scanning process, the RSSI of each neighbor BS. The MS draws or updates the scatter diagrams of each pair of MS BS neighbor to obtain the estimated regression line. Based on the estimated regression line the MS predict the future RSSI value which is the response of $t=t_{c}+\Delta t$, where $t_{c}$ is the current time and $\Delta t$ is the prediction interval. $\left((R S S I)_{j}\right)_{i}$, is the Received Signal Strength Indicator of BSj at time ti;

$\mathrm{i}=1, \ldots \ldots . ., \mathrm{T}$ where $\mathrm{T}$ is the number of scan iteration authorized for the MS and fixed by the serving BS.

$(R S S I)_{i}=\alpha+\beta t_{i}+\varepsilon_{i} \Rightarrow \varepsilon_{i}=(R S S I)_{i}-\alpha+\beta t_{i}$

The linear regression model chooses the estimator values of $\alpha$ and $\beta$ that minimize the value $\sum_{i=1}^{T} \varepsilon_{i}^{2} \quad \sum_{i=1}^{T} \varepsilon_{i}^{2}=\sum_{i=1}^{T}\left[(R S S I)_{i}-\alpha-\beta t_{i}\right]^{2}$, according to the regression model the estimator of $\alpha$ and $\beta$ are given by:

$$
\left\{\begin{array}{l}
\alpha=\overline{(R S S I)-\overline{\beta t}} \\
\beta=\frac{\sum_{i=1}^{T}\left[\left(t_{i}-\bar{t}\right) \times\left((R S S I)_{i}-(\overline{R S S I})\right)\right]}{\sum_{i=1}^{T}\left(t_{i}-\bar{t}\right)^{2}}
\end{array}\right.
$$

$(\overline{R S S I})=\frac{\sum_{i=1}^{T}(R S S I)_{i}}{T} \quad$ and $\quad \bar{t}=\frac{\sum_{i=1}^{T} t_{i}}{T}$

In our algorithm we initiate four variables:

Scth: Scan threshold,

Hoth : Handover threshold,

$\Delta t$ : Prediction interval,

$\mathrm{H}$ : hysteresis margin.

The MS is expected to initiate handover after $\Delta t$ if the predicted RSSI is less than Hoth

The (RSSI) Serving and (RSSI) Target are respectively the BS serving and the target Received Signal Strength Indicator.

In order to avoid the ping pong effects and control the handover decision we use a handover hysteresis margin between the serving and target BSs [13][10]. The decision is made when:

(RSSI) Target $>$ Hoth and (RSSI) Target - (RSSI) Serving $>\mathrm{H} \mathrm{H}$ in (dB).

The prediction based handover algorithm is detailed in figure 3 . 


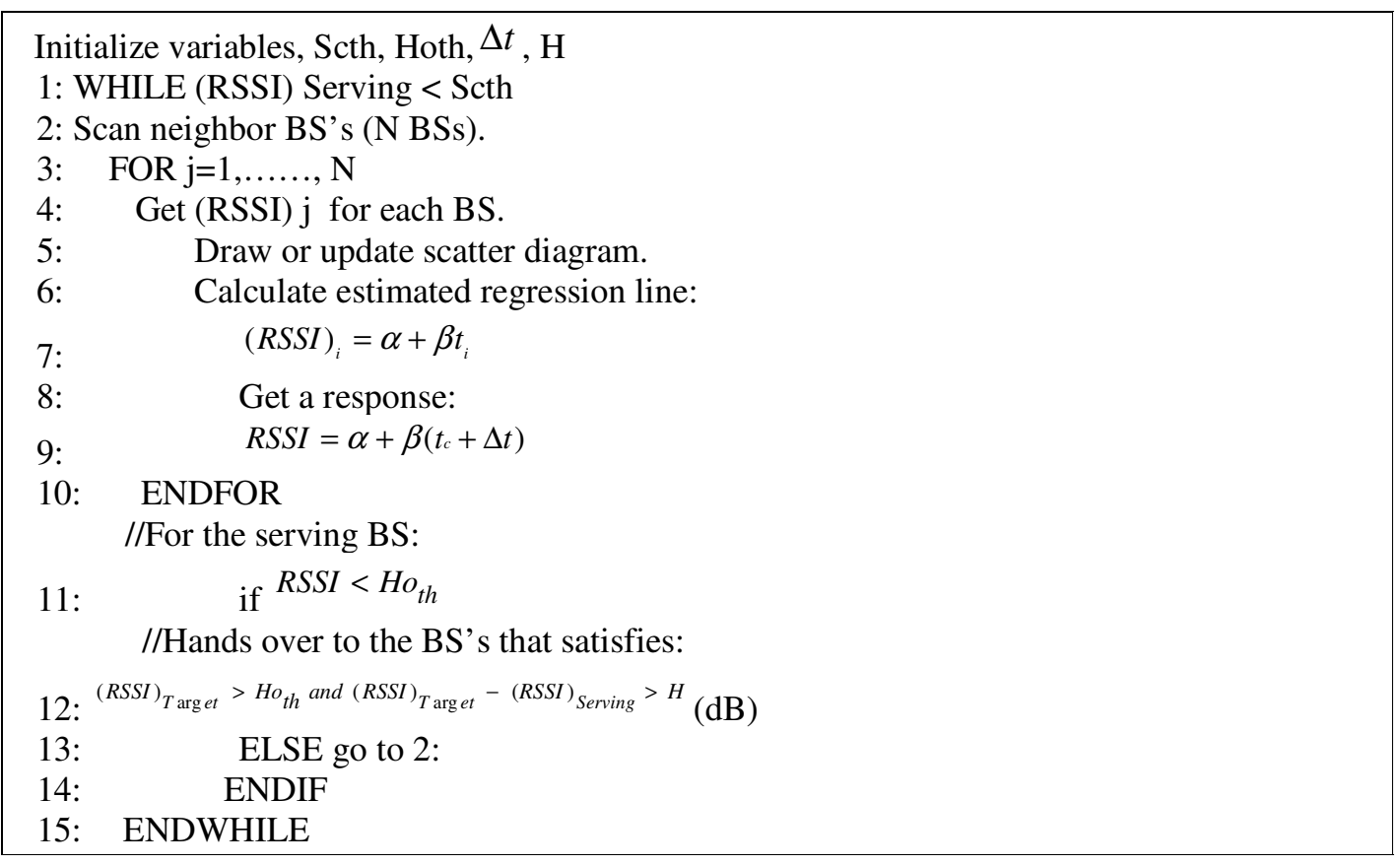

Figure.3. Prediction based handover Algorithm.

\section{PERFORMANCE EVALUATION.}

The main objective of the Prediction based handover Algorithm is to reduce the handover latency and the packets lost during the handover operation. The total handover latency can be shortened by the amount of $\Delta t$.

\section{Simulation handover scenario.}

The simulations were performed using the publicly free available NS-2 simulator and the NIST packages of WiMAX and mobility [14]. In our scenario, we use three BSs located in a triangular form. The coverage area of each one is $2 \mathrm{~km}$. The MS moves from the center of the serving base station (BS1) toward the center of the target base station (BS2). The MS can monitor the RSSI of the third BS (BS3) as showed in the figure 3.

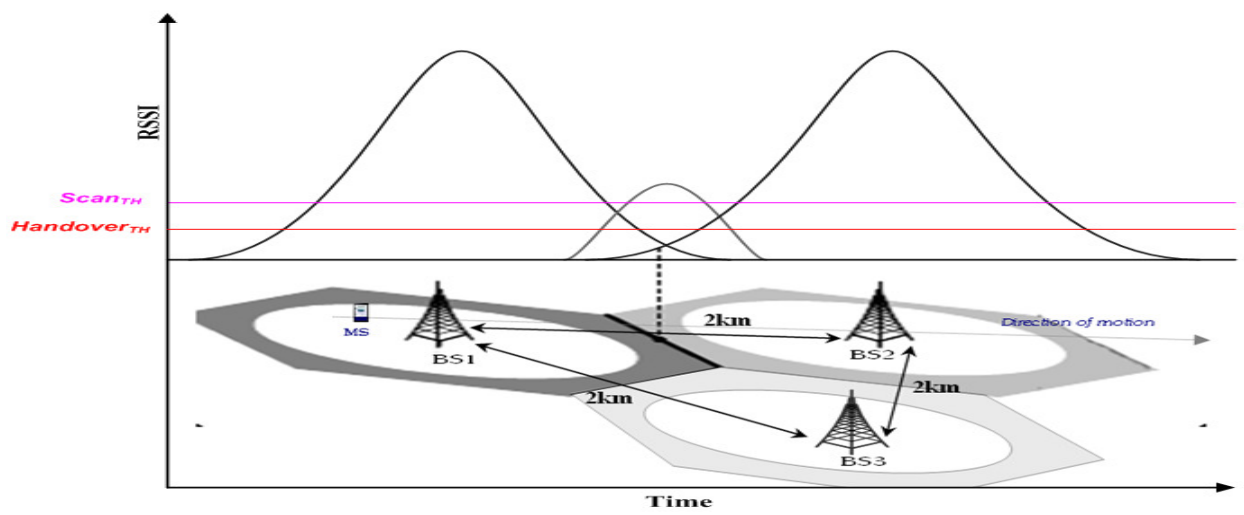

Figure.3. Simulation handover scenario. 
After the adjustments of NS-2 and WiMAX-module parameters, the simulation was done with the following parameters: MS speeds of $120 \mathrm{~km} / \mathrm{h}$, MAC frame length of $5 \mathrm{~ms}$, neighbor scan duration and interleaving interval of $100 \mathrm{~ms}$. Prediction of future RSSI $\Delta t=100 \mathrm{~ms}$, scan threshold and Handover threshold are respectively of $15 \%$ and $10 \%$ of the maximum RSSI and a handover hysteresis margin of $-3 \mathrm{~dB}$. The allover of the simulation duration was initiated at 100s. In the next section, we compare the simulation results in two scenarios: one with prediction handover algorithm and other without prediction algorithm.

\section{Simulation Result}

In this section the performance evaluation of the proposed prediction handover algorithm is presented in terms of total handover latency, number of packets loss and the packets arrival delay. We compare the results of two scenarios, one with predictive algorithm and another without prediction. We focus on the handover time interval in all our figures.

The figure 4 shows the throughput evolution during the handover time interval. We observe that, in the non predictive assistant scenario, the handover process begins at 56.725 seconds since the beginning of simulation and finish at 57.65. The total time process handover is $950 \mathrm{~ms}$. On the contrary in the assisted scenario the handover begins at $56.75 \mathrm{~s}$ and ends at $57.6 \mathrm{~s}$. The handover latency is about $850 \mathrm{~ms}$. We observe that the total handover latency was reduced by $75 \mathrm{~ms}$.

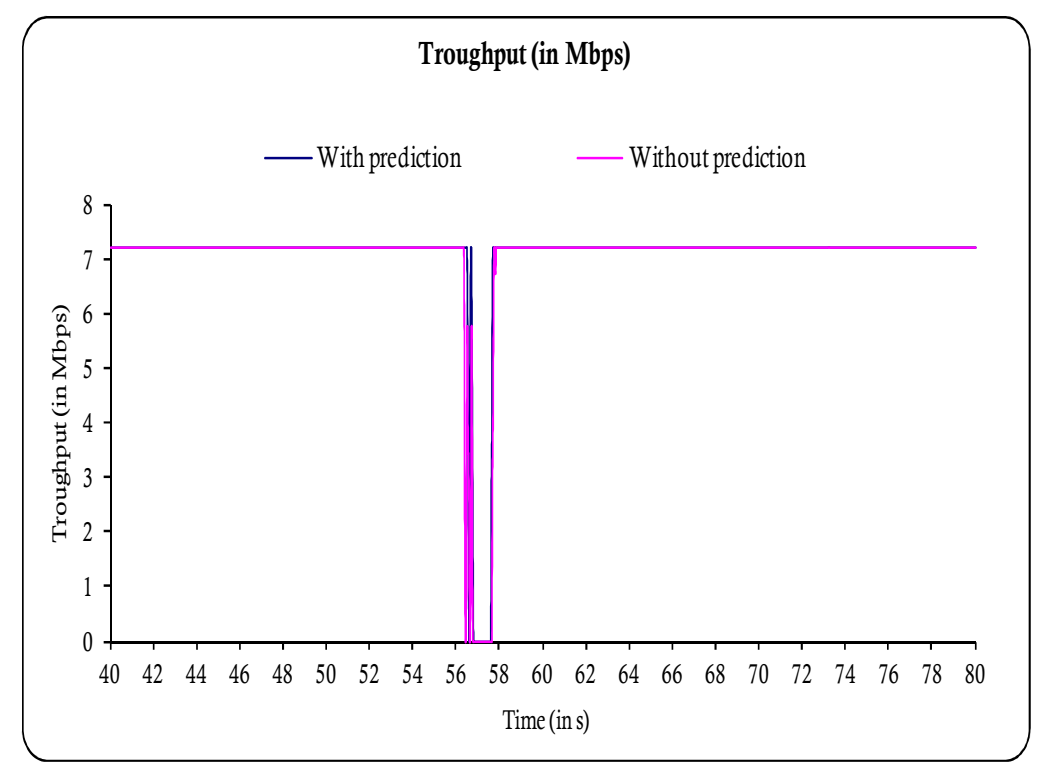

Figure.4. Throughput vs time for the two scenarios.

As shown in figure 5 the number of the packets loss was also reduced. In the first scenario the total number of the packet lost is 5200 packets, but in the second scenario the total number of the packet lost is 3200 packs, that make a benefit of 2000 packets. 
International Journal of Distributed and Parallel Systems (IJDPS) Vol.3, No.3, May 2012

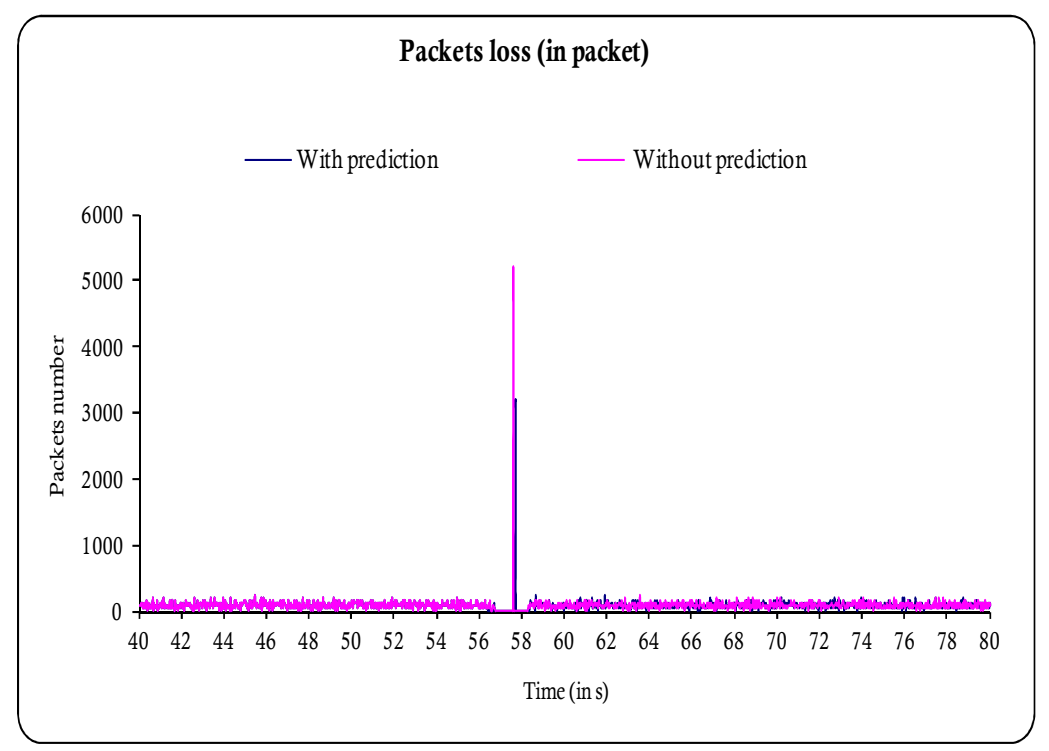

Figure.5. Number of packets loss vs time for the two scenarios.

In the figure 6 we also observe that the packets arrival delay was also reduced. In the first scenario the means packets arrival delay after handover was $3.34253473 \mathrm{~ms}$ but in the second scenario is $3.35143075 \mathrm{~ms}$ that make a benefit of $0.00889603 \mathrm{~ms}$.

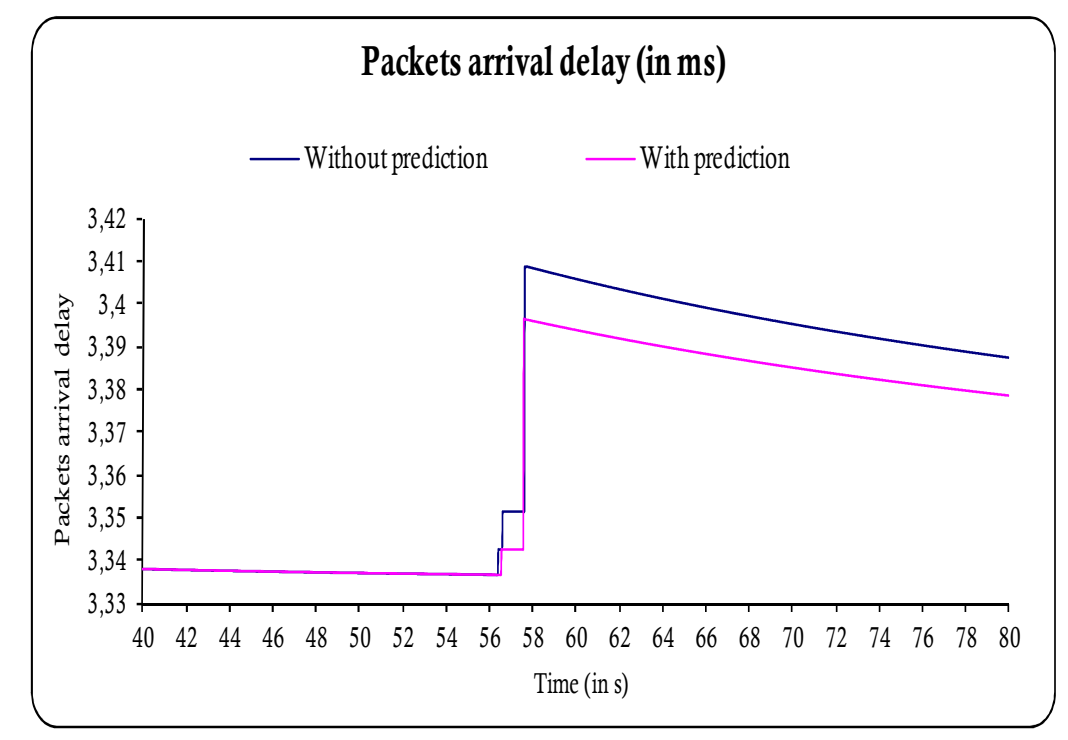

Figure.6. Packets arrival delay vs time for the two scenarios.

\section{CONCLUSIONS.}

In this study, we use the linear regression model to build a predictive hard handover algorithm that predict the Received Signal Strength Indicator value and advertize the MS to trigger the scanning procedure and the handover process operations reducing so the total handover latency and packets loss rate. The numerical analysis and simulation results show that the proposed method significantly reduces the handover latency and the packets loss rate. We also simulate 
International Journal of Distributed and Parallel Systems (IJDPS) Vol.3, No.3, May 2012

three performance parameters for the handover latency in the mobile WiMAX based networks to improve our algorithm. Theoretically the predictive hard handover algorithm introduced here, reduce the total handover latency of $100 \mathrm{~ms}$. In the simulation results we observe that the handover latency was reduced by $75 \mathrm{~ms}$. The number of packets loss during the handover process and the packets arrival delay was also reduced.

\section{REFERENCES}

[1] Institute of Electrical and Electronics Engineers, "IEEE Standard for Local and Metropolitan Area Networks - Part 16: Air Interface for Fixed Broadband Wireless Access Systems, Amendment 2: Physical and Medium Access Control Layers for Combined Fixed and Mobile Operation in Licensed Bands", IEEE Std 802.16e, Feb., 2006.

[2] Jeffrey G.Andrews, Arunabha Ghosh and Rias Muhamed Fundamentals of WiMAX, Understanding Broadband Wireless Networking. Prentice Hall. Communications Engineering and emerging Technologies Series. pp 52.

[3] Mobile WiMAX A Systems Approach to Understanding IEEE 802.16m Radio Access Technology. Sassan Ahmadi. Academic Press 2011 Elsevier Inc. pp 183.

[4] I. Akyildiz and W. Wang, "The Predictive User Mobility Profile Framework for Wireless Multimedia Networks," IEEE/ACM Trans. Networking, Vol. 12, No. 6, pp. 1021-1035,Dec. 2004.

[5] Z. Becvar and J. Zelenka, Handovers in the Mobile WiMAX, In Research in Telecommunication Technology 2006 - Proceedings [CD-ROM]. Brno: Vysok'e ǔcen’e technick'e v Brn`e, 2006, Vol. I, pp. 147-50.

[6] H. Fattah, H. Alnuweiri; A New Handover Mechanism for IEEE802.16e Wireless Networks, Wireless communications and Mobile Computing Conference, 2008. IW CMC'08. International, August 2008.

[7] J. Choi, N. Hao, S. Yoo; Fast groups canning scheme with dynamic neighbor base station list in IEEE802.16e networks $8^{\text {th }}$ Conference on Computer and Information Technology (CIT ), July 2008.

[8] R. Hsieh et al., "S-MIP: A Seamless Handoff Architecture for Mobile IP," IEEE INFOCOM03, pp. 1774-1784, Apr. 2003.

[9] Richard Rouil and Nada Glomie, "Adaptive Channel Scanning for IEEE 802.16e", IEEE MILCOM 2006, pp. 1-6, 2006.

[10] WIMAX SECURITY AND QUALITY OF SERVICE AN END-TO-END PERSPECTIVE Edited by Seok-Yee Tang Peter Müller and Hamid R. Sharif ( 2010 John Wiley \& Sons, Ltd. pp 186 and 224

[11] WiMAX Standards and Security. "Mobility Support for IEEE 802.16e System" HyunHo Choi and Dong-Ho Cho, 2008. Edited by SYED AHSON MOHAMMAD ILYAS pp 114.

[12] C. Tseng et al., "Topology-Aided Cross-Layer Fast Handoff Designs for IEEE 802.11/Mobile IP Environments," IEEE Commun. Mag., Vol.12, Iss. 12, pp. 156-163, Dec. 2005.

[13] Handover performance in the mobile WiMAX Networks Yongxue $Y \boldsymbol{u}$ University of South Florida. pp 60-63.

[14] http://www.nist.gov/itl/antd/emntg/ssm_tools.cfm 